\title{
Changes in Surface Convexity and Topology Caused by Distortions of Stereoscopic Visual Space
}

\author{
Gregory Baratoff $^{1}$ and Yiannis Aloimonos ${ }^{2}$ \\ 1 Dept. of Neural Information Processing, University of Ulm, \\ 89069 Ulm, GERMANY, \\ barat of $f$ eneuro.informatik.uni-ulm.de, \\ 2 Center for Automation Research, University of Maryland, \\ College Park, MD 20742, USA, \\ yiannis@cfar.umd.edu
}

\begin{abstract}
We introduce the notion of a distorted reconstruction from two views, which need not satisfy the epipolar constraint. It can be computed from point correspondences and from a possibly inexact estimate of the stereo configuration. Thus, this scheme avoids the often costly and unstable minimization procedure for establishing the epipolar constraint, at the cost of introducing non-linear distortions. As a consequence, the convexity and topology of curves and surfaces can be changed.

The distorted reconstruction is related to the original scene structure by $a$ quadratic Cremona transformation of space. By analyzing the distortion of curves and surfaces geometrically in terms of the singular elements of the associated Cremona transformation, we show that severe distortions are present particularly in the vicinity of the camera centers, thereby indicating that their consideration is of particularly high relevance for near regions of the stereo rig. Our main technical contribution is the derivation of the exact criteria governing changes in surface convexity and topology.
\end{abstract}

\section{Introduction}

One of the central tasks of a binocular (or stereoscopic) vision system is to reconstruct the scene from the projections of environmental structures onto the two views. The reconstruction computation is based on two pieces of information :

- a set of corresponding points (or lines) in the two views.

- the stereo configuration, i.e. the relative positions and orientations of the two cameras, and their internal parameters.

Corresponding points are usually obtained by a matching procedure based on purely visual attributes, whereas the stereo configuration can be obtained from either visual or by non-visual sources of information. 


\subsection{Reconstruction and the Epipolar Constraint}

By whatever means it is obtained, the stereo configuration needs to be known exactly if the resulting reconstruction is to be veridical, i.e. if metric properties such as distances and angles are to be preserved. Unfortunately, obtaining the exact stereo configuration can be problematic. Estimates of the stereo configuration based on non-visual information are often not accurate enough. Furthermore, they are usually inconsistent with the epipolar constraint, which expresses the fact that corresponding rays issued by the two cameras should intersect in space. In order to exploit visual information, the cameras have to be calibrated individually, and their relative orientation has to be found, with both procedures in general requiring a non-linear minimization[16]. Especially in the presence of noise these computations are plagued by instabilities, and convergence can not in general be guaranteed.

Partial relief comes from recent advances in the field of computer vision showing that a projective reconstruction can still be obtained even if the internal parameters of both cameras are unknown[7]. A projective reconstruction is a projective transformation of the original scene structure, i.e. it is linear when expressed in terms of homogeneous coordinates. Furthermore, a projective reconstruction satisfies the epipolar constraint. A more practical reason for its attractiveness is the fact that a projective representation is adequate for a variety of tasks, such as recognition and navigation[5,11].

But, just as is the case for the calibration of the stereo rig in the metric case, computing a projective reconstruction can be an unstable process[9]. In both cases the instability arises from the attempt to satisfy the epipolar constraint. The question we asked ourselves is whether this step could be avoided, and if so, what the nature of the resulting, necessarily non-linear, distortions would be.

\subsection{Distorted Reconstruction}

The reconstructed position of a point in space is usually defined as the intersection of the two corresponding rays. Therefore, the reconstruction is in general not even defined unless the epipolar constraint is satisfied. We remedy this by extending the definition of the reconstruction to point correspondences which are incompatible with the epipolar constraint. We call this new reconstruction a distorted reconstruction. There are many different ways of defining it, but we choose here a simple one that is at once geometrically intuitive and algebraically tractable : instead of the intersection of the two rays, the distorted reconstruction of a point is defined as the intersection of one of the rays and a plane containing the other ray. ${ }^{1}$ This kind of distorted reconstruction can be termed a reconstruction with a dominant camera. The specific instance we will be using is shown in Figure 1, where the left camera is considered the dominant one, by virtue of providing the ray, whereas the right camera only provides a plane (the one defined by the $x$-coordinate).

\footnotetext{
${ }^{1}$ One alternative is the midpoint of the shortest segment connecting the two rays.
} 
We do not claim originality for the form of the definition of the distorted reconstruction. Even in traditional approaches the reconstruction is sometimes computed in this manner, however only once an admissible stereo configuration has been found. Our approach represents, rather, a shift in emphasis : we consider the distorted reconstruction a legitimate reconstruction that is worthy of study. In going with this view, we take as the object of our analysis not the error in reconstruction, i.e. the difference between distorted and true structure, but the transformation relating the two. We call the transformation between the true and the distorted reconstruction of a scene the shape distortion transformation, or shape distortion for short. This also makes it more evident that we are considering the systematic relation between the two reconstructions arising from the use of an incorrect estimate of the stereo configuration, and not random errors due to image noise or matching errors. We have argued elsewhere[1] that both sources of error need to be considered, but in this article we concentrate on systematic errors.

The distorted reconstruction from image correspondences involves what we call the apparent stereo configuration, different from the true stereo configuration used in the projection of the scene onto the cameras. Furthermore, we refer to an apparent space, in which objects live that were reconstructed using the apparent configuration, and a true space, in which objects live that were reconstructed using the true configuration. In order to distinguish objects from the different spaces, we will always use primed symbols (e.g. $\mathbf{X}^{\prime}, \mathbf{m}^{\prime}$ ) to refer to objects in the apparent space.

\subsection{Related Work}

The framework of distortions was introduced by [6] in the case of calibrated cameras, and under the assumption of infinitesimal motion. It was shown there that quadratic terms appeared in the reconstruction. Here, we show that the shape distortion transformation in the case of discrete displacements is a quadratic Cremona transformation for arbitrarily positioned and oriented, uncalibrated cameras. Thus, the framework applies to distortions of metric as well as projective reconstructions. Our application of global properties of Cremona transformations to qualitatively explain the shape distortion is novel; we first used it to study the distortions in the planar case [2,3]. Quadratic distortions have been observed by photogrammetrists[13] in certain stereoscopes. In the Computer Vision literature, (plane) quadratic transformations have been used to describe the mapping between corresponding points in two image planes (e.g. [10]), but have not been applied in the analysis of transformations of reconstructed space.

\section{The Shape Distortion}

\subsection{Double Algebra}

In this and in the following sections we utilize the framework of Double Algebra[4] to derive and analyze the shape distortion. Double algebra was first used 
in Computer Vision by Svensson [15], and has gained interest in recent years. Specifically, we employ the double algebra of $\mathcal{R}^{4}$ to represent points, lines, and planes in projective space $\mathcal{P}^{3}$ by (linear) subspaces of $\mathcal{R}^{4}$ in terms of homogeneous vectors. Two homogeneous vectors $\mathbf{a}$ and $\mathbf{b}$ that are equal up to a non-zero scale factor $k$, i.e. $\mathbf{a}=k \mathbf{b}$ represent the same subspace, and we write $\mathbf{a} \cong \mathbf{b}$ when we do not wish to mention the scale factor.

Double Algebra provides the join and meet operators $\vee$ and $\wedge$ for combining subspaces in various ways. For example, one writes :

$-\mathbf{l}=\mathbf{A} \vee \mathbf{B}$ for the line $\mathbf{1}$ formed by joining the two points $\mathbf{A}$ and $\mathbf{B}$,

$-\mathbf{p}=\mathbf{l} \vee \mathbf{C}$ for the plane $\mathbf{p}$ formed by joining $\mathbf{l}$ to a third point $\mathbf{C}$,

- $\mathbf{D}=\mathbf{k} \wedge \mathbf{p}$ for the point $\mathbf{D}$ in which the line $\mathbf{k}$ meets the plane $\mathbf{p}$.

The meet and the join are both anti-symmetric, i.e. $\mathbf{A} \vee \mathbf{A}=0$, expressing the fact that the join of a point with itself is undefined, and $\mathbf{p} \wedge \mathbf{p}=0$, expressing the fact that the meet of a plane with itself is undefined. They are also linear in both arguments. Underlying the join and meet operators is the notion of a bracket, which is a coordinate-free abstraction of a determinant. In $\mathcal{P}^{3}$, the bracket is defined for any sequence of four points $\left\{\mathbf{X}_{i}\right\}_{i=1}^{\mathbf{4}}$, and is written as $\left[\begin{array}{llll}\mathbf{X}_{1} & \mathbf{X}_{2} & \mathbf{X}_{3} & \mathbf{X}_{4}\end{array}\right]$. It vanishes when the four points are coplanar, which happens when the $\mathbf{X}_{i}$ are linearly dependent. We will also use the shortened form $\left[\mathbf{m}, \mathbf{X}_{4}\right]$ where $\mathbf{m}=\mathbf{X}_{1} \vee \mathbf{X}_{2} \vee \mathbf{X}_{3}$ is a plane.

\subsection{Camera Geometry}

We use the pinhole camera model, and use a representation of the camera in terms of its plane star [2]. ${ }^{2}$ Let $\mathbf{m}_{1}, \mathbf{m}_{2}, \mathbf{m}_{3}$ be the base planes of the camera. ${ }^{3}$ From now on we use the terms plane star and camera interchangeably. The center of projection of a camera is given by $\mathbf{O}_{M}=\mathbf{m}_{1} \wedge \mathbf{m}_{2} \wedge \mathbf{m}_{3}$ as the point of intersection of the three base planes. We define the projection of a point $\mathbf{X}$ onto the camera $M$ as $\left(\left[\mathbf{m}_{1}, \mathbf{X}\right]\left[\mathbf{m}_{2}, \mathbf{X}\right]\left[\mathbf{m}_{3}, \mathbf{X}\right]\right)^{T}$.

An alternative way to look at a camera is to interpret it as a line star, i.e. the collection of lines that pass through the camera center. Given a plane star $M$, we define the associated line star matrix $L_{M}=\left(\mathbf{x}_{M} \mathbf{y}_{M} \mathbf{z}_{M}\right)$, where $\mathbf{x}_{M}=\mathbf{m}_{2} \wedge \mathbf{m}_{3}, \mathbf{y}_{M}=\mathbf{m}_{3} \wedge \mathbf{m}_{1}, \mathbf{z}_{M}=\mathbf{m}_{1} \wedge \mathbf{m}_{2}$ are the base lines.

Given a camera $M$, we define the $x$-plane $\mathbf{m}_{x}(\mathbf{X})$, the $y$-plane $\mathbf{m}_{y}(\mathbf{X})$, and the ray $\mathbf{l}_{M}(\mathbf{X})$ of $M$ through a point $\mathbf{X}$ as follows :

$$
\begin{aligned}
& \mathbf{m}_{x}(\mathbf{X}) \doteq-\mathbf{y}_{M} \vee \mathbf{X}=\left[\mathbf{m}_{1}, \mathbf{X}\right] \mathbf{m}_{3}-\left[\mathbf{m}_{3}, \mathbf{X}\right] \mathbf{m}_{1} \\
& \mathbf{m}_{y}(\mathbf{X}) \doteq \mathbf{x}_{M} \vee \mathbf{X}=\left[\mathbf{m}_{2}, \mathbf{X}\right] \mathbf{m}_{3}-\left[\mathbf{m}_{3}, \mathbf{X}\right] \mathbf{m}_{2} \\
& \mathbf{l}_{M}(\mathbf{X}) \doteq \mathbf{O}_{M} \vee \mathbf{X}=\left[\mathbf{m}_{1}, \mathbf{X}\right] \mathbf{x}_{M}+\left[\mathbf{m}_{2}, \mathbf{X}\right] \mathbf{y}_{M}+\left[\mathbf{m}_{3}, \mathbf{X}\right] \mathbf{z}_{M}
\end{aligned}
$$

$\overline{2}$ A plane star is the 2-parameter family of planes through a common point. Any plane of the plane star can be generated as a linear combination of three base planes.

${ }^{3}$ The $3 \times 4$ matrix commonly used to represent a projective camera is given in terms of the plane star representation as $-M^{T}$, where $M=\left(\begin{array}{lll}\mathbf{m}_{1} & \mathbf{m}_{2} & \mathbf{m}_{3}\end{array}\right)$. See [2]. 
Stereo Geometry Let $C$ and $D$ be two cameras. Then, a point $\mathbf{X}$ can be reconstructed by computing the meet of, say, the ray $\mathbf{l}_{C}(\mathbf{X})$ of $C$ with the $x$ plane $\mathbf{d}_{x}(\mathbf{X})$ of $D$, i.e. $\mathbf{X} \cong \mathbf{l}_{C}(\mathbf{X}) \wedge \mathbf{d}_{x}(\mathbf{X})$. (See Fig. 1.) Note that there are other possible reconstructions (e.g. replace $\mathbf{d}_{x}(\mathbf{X})$ by $\mathbf{d}_{y}(\mathbf{X})$ ), but they are all equivalent if the apparent and true cameras are identical.

\subsection{Distorted Reconstructions of Rays, Planes, and Points}

In a distorted reconstruction we have an apparent stereo configuration, given by the pair of cameras $C^{\prime}$ and $D^{\prime}$, which are in general not identical with the true cameras $C$ and $D$. We first define the distorted reconstructions of camera planes and rays (by modification of equations (1)-(3)) :

$$
\begin{aligned}
\mathbf{m}_{x}^{\prime}(\mathbf{X}) & =\left[\mathbf{m}_{1}, \mathbf{X}\right] \mathbf{m}_{3}^{\prime}-\left[\mathbf{m}_{3}, \mathbf{X}\right] \mathbf{m}_{1}^{\prime} \\
\mathbf{m}_{y}^{\prime}(\mathbf{X}) & =\left[\mathbf{m}_{2}, \mathbf{X}\right] \mathbf{m}_{3}^{\prime}-\left[\mathbf{m}_{3}, \mathbf{X}\right] \mathbf{m}_{2}^{\prime} \\
\mathbf{l}_{M}^{\prime}(\mathbf{X}) & =\left[\mathbf{m}_{1}, \mathbf{X}\right] \mathbf{x}_{M}^{\prime}+\left[\mathbf{m}_{2}, \mathbf{X}\right] \mathbf{y}_{M}^{\prime}+\left[\mathbf{m}_{3}, \mathbf{X}\right] \mathbf{z}_{M}^{\prime}
\end{aligned}
$$

These we call direct distorted planes and rays. They live in apparent space, whereas $\mathbf{X}$ lives in true space. Similarly, we also define the reverse distorted planes and rays for a point $\mathbf{X}^{\prime}$ in the apparent space :

$$
\begin{aligned}
\mathbf{m}_{x}\left(\mathbf{X}^{\prime}\right) & =\left[\mathbf{m}_{1}^{\prime}, \mathbf{X}^{\prime}\right] \mathbf{m}_{3}-\left[\mathbf{m}_{3}^{\prime}, \mathbf{X}^{\prime}\right] \mathbf{m}_{1}^{\prime} \\
\mathbf{m}_{y}\left(\mathbf{X}^{\prime}\right) & =\left[\mathbf{m}_{2}^{\prime}, \mathbf{X}^{\prime}\right] \mathbf{m}_{3}-\left[\mathbf{m}_{3}^{\prime}, \mathbf{X}^{\prime}\right] \mathbf{m}_{2} \\
\mathbf{1}_{M}\left(\mathbf{X}^{\prime}\right) & =\left[\mathbf{m}_{1}^{\prime}, \mathbf{X}^{\prime}\right] \mathbf{x}_{M}+\left[\mathbf{m}_{2}^{\prime}, \mathbf{X}^{\prime}\right] \mathbf{y}_{M}+\left[\mathbf{m}_{3}^{\prime}, \mathbf{X}^{\prime}\right] \mathbf{z}_{M}
\end{aligned}
$$

These entities live in true space, whereas $\mathbf{X}^{\prime}$ lives in apparent space. The direct distorted reconstruction of a point $\mathbf{X}$ is given by $\mathbf{l}_{C}^{\prime}(\mathbf{X}) \wedge \mathbf{d}_{x}^{\prime}(\mathbf{X})$. It is illustrated in Figure 1. We have the following result :

Theorem 1. (Space Shape Distortion)

Let $(C, D)$ be the true stereo configuration, and $\left(C^{\prime}, D^{\prime}\right)$ the apparent one. Then the shape distortion transformation $\mathbf{T}(\mathbf{X}) \doteq \mathrm{l}_{C}^{\prime}(\mathrm{X}) \wedge \mathrm{d}_{x}^{\prime}(\mathbf{X})$ is a quadratic space Cremona transformation. Its reverse is $\mathbf{T}^{\prime}\left(\mathbf{X}^{\prime}\right) \doteq \mathbf{l}_{C}\left(\mathbf{X}^{\prime}\right) \wedge \mathbf{d}_{x}\left(\mathbf{X}^{\prime}\right)$.

Proof. That $\mathbf{T}$ is quadratic in $\mathbf{X}$ follows from the fact that both $\mathbf{l}_{C}^{\prime}(\mathbf{X})$ and $\mathbf{d}_{x}^{\prime}(\mathbf{X})$ are linear in $\mathbf{X}$, and from the linearity of the meet. The proof that $\mathbf{T}^{\prime}$ is indeed the reverse transformation of $\mathbf{T}$ can be found in [2].

\section{Global Analysis of the Shape Distortion}

In this section we review some established results on Cremona transformations [12], and then apply them to the shape distortion transformation. 


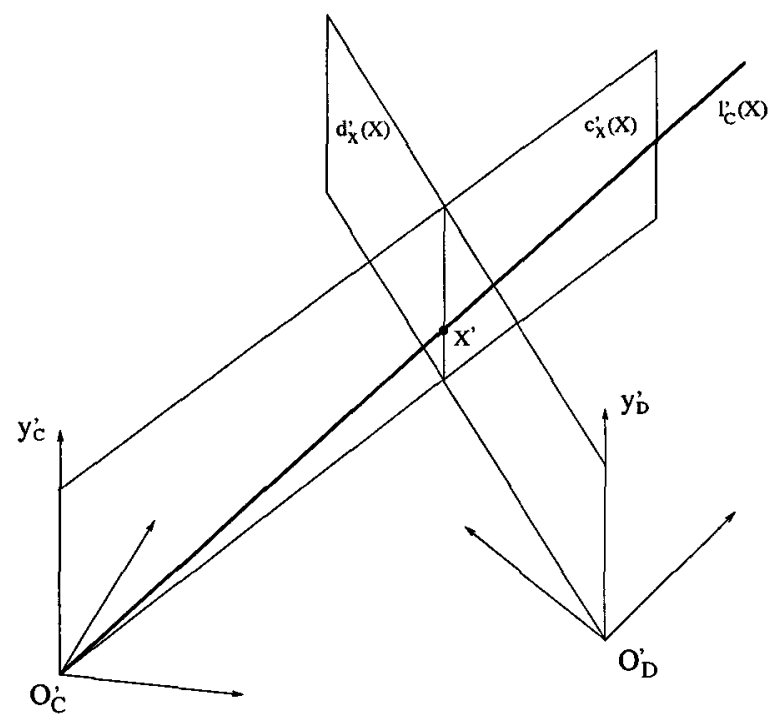

Fig. 1. Distorted reconstruction as the intersection of the distorted ray $\mathbf{l}_{C}^{\prime}(\mathbf{X})=$ $\mathbf{c}_{x}^{\prime}(\mathbf{X}) \wedge \mathbf{c}_{y}^{\prime}(\mathbf{X})$ of camera $C$ with the distorted $x$-plane $\mathbf{d}_{x}^{\prime}(\mathbf{X})$ of camera $D$.

\subsection{Cremona Transformations}

A Cremona transformation is a birational transformation, i.e. a rational transformation whose inverse is also rational. This means that Cremona transformations are one-to-one mappings in general. A trivial example of a Cremona transformation is a collineation. It is one-to-one everywhere. However, for transformations of higher order there always exist exceptional points for which the transformation is not one-to-one.

A Cremona transformation $\mathbf{T}$ in $\mathcal{P}^{3}$ can be represented by a vector of 4 homogeneous polynomials $\mathcal{T}_{i}$ :

$$
\mathbf{T}: \mathbf{X} \mapsto \mathbf{X}^{\prime}=\left(\mathcal{T}_{1}(\mathbf{X}) \mathcal{T}_{2}(\mathbf{X}) \mathcal{T}_{3}(\mathbf{X}) \mathcal{T}_{4}(\mathbf{X})\right)^{T} .
$$

Cremona transformations have the property that the polynomials $\mathcal{T}_{i}$ all have the same degree; this is called the order of the transformation.

Since a Cremona transformation is a one-to-one mapping almost everywhere, its inverse $\mathbf{T}^{-1}$ is defined almost everywhere. One usually works instead with the reverse transformation $\mathbf{T}^{\prime}$, which is equal to the inverse up to scale, i.e. $\mathbf{T}^{\prime}(\mathbf{T}(\mathbf{X})) \cong \mathbf{T}^{-1}(\mathbf{T}(\mathbf{X}))=\mathbf{X}$. Since we are dealing with transformations between homogeneous coordinates, the scale does not affect the mapping of the non-homogeneous coordinates as long as it does not vanish. We write the reverse transformation as

$$
\mathbf{T}^{\prime}: \mathbf{X}^{\prime} \mapsto \mathbf{X}=\left(\mathcal{T}_{1}^{\prime}\left(\mathbf{X}^{\prime}\right) \mathcal{T}_{2}^{\prime}\left(\mathbf{X}^{\prime}\right) \mathcal{T}_{3}^{\prime}\left(\mathbf{X}^{\prime}\right) \mathcal{T}_{4}^{\prime}\left(\mathbf{X}^{\prime}\right)\right)^{T}
$$

It has the same order as $\mathbf{T}$, since a Cremona transformation and its reverse always have the same order [12]. 
Singular Elements The exceptional elements at which $\mathbf{T}$ is not one-to-one are simply those at which $\mathbf{T}(\mathbf{X})=\mathbf{0}$, i.e. where all polynomials $\mathcal{T}_{i}(\mathbf{X})$ vanish. The set of such points $\mathbf{X}$ forms the base elements of $\mathbf{T}$. For a quadratic space Cremona transformation, the base is made up of a base point $\mathbf{B}_{1}$, and a base conic $\mathbf{b}$. We will see that for our purposes the special case of a degenerate base conic $\mathbf{b}$ will be important; in this case $\mathbf{b}$ splits into two intersecting lines $\mathbf{b}_{2}$ and $\mathbf{b}_{3}$. Since $\mathbf{T}^{\prime}$ is of the same order as $\mathbf{T}$, its base has exactly the same structure as that of $\mathbf{T}$. We denote its base elements by the corresponding primed quantities $\mathbf{B}_{1}^{\prime}$ and $\mathbf{b}^{\prime}$, respectively $\mathbf{b}_{2}^{\prime}, \mathbf{b}_{3}^{\prime}$.

A further set of elements, which in a sense is dual to the base elements, is also relevant for the characterization of Cremona transformations. These are the fundamental elements. With a quadratic Cremona transformation is associated a fundamental plane $\mathbf{f}_{1}$, which is the plane containing the base conic $\mathbf{b}$, and a cone of fundamental lines, each generating line of which is formed by connecting the base point $\mathbf{B}_{1}$ to a point on the base conic $\mathbf{b}$. In the degenerate case of interest to us, $\mathbf{f}_{\mathbf{1}}$ is spanned by $\mathbf{b}_{2}$ and $\mathbf{b}_{3}$, and the cone of fundamental lines splits into two planes $\mathbf{f}_{2}$ and $\mathbf{f}_{3}$ of fundamental lines. This configuration is illustrated in Figure 2. The fundamental elements of $\mathbf{T}^{\prime}$ can be defined in an analogous way, and will be referred to by the corresponding primed quantities $f_{i}^{\prime}$.

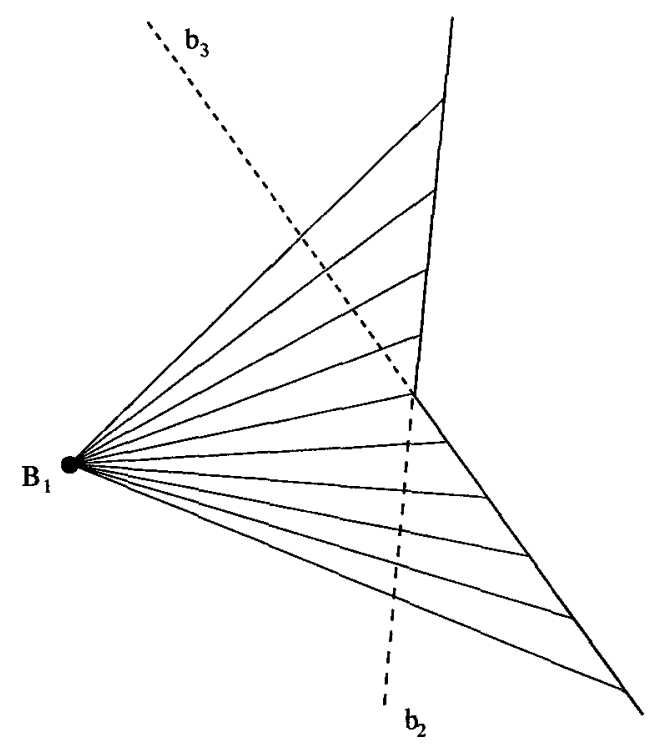

Fig. 2. Base elements of a quadratic space Cremona transformation with degenerate base conic.

The reason that we single out the fundamental elements is that $\mathbf{T}$ maps its fundamental elements onto the base elements of $\mathbf{T}^{\prime}$, and vice versa. Specifically, $\mathbf{T}$ maps $\mathbf{f}_{1}$ onto $\mathbf{B}_{1}^{\prime}$, and each fundamental line of $\mathbf{f}_{2}\left(\mathbf{f}_{3}\right)$ to a point of $\mathbf{b}_{2}\left(\mathbf{b}_{3}\right)$. 
Proposition 2. (Topology Changes) The topology of a surface changes under the shape distortion iff the surface meets one of the following conditions :

- it intersects the fundamental plane $\mathbf{f}_{1}$.

- it intersects one of the planes of fundamental lines $\mathbf{f}_{2}$ and $\mathbf{f}_{3}$ in such a way that it contains at least two distinct points of the same fundamental line.

Proof. From section 3.1, we know that all points of a given fundamental element map to the same point. Thus, if a surface contains two distinct points belonging to the same fundamental element, the image of the surface will pass twice through the same point, thus creating a self-intersection, clearly a change of topology. A proper intersection with $\mathbf{f}_{1}$ yields an entire curve, whereas an intersection with $\mathbf{f}_{2}$ or $\mathbf{f}_{3}$ does not necessarily intersect any of the fundamental lines within these planes twice. Thus, the additional requirement for $\mathbf{f}_{2}$ and $\mathbf{f}_{3}$.

\subsection{Restriction to Preserved Planes}

The space shape distortion is a complicated transformation. We could simplify the analysis if we considered the restriction of the space shape distortion to planes. However, we can not in general do this, since a plane maps into a (web) quadric, and not into a plane. A plane-to-plane mapping only exists if the web quadric is degenerate. Luckily, it turns out that there are enough of these degenerate quadrics for the purposes of our analysis. The next proposition tells us which ones they are :

Proposition 3. Let $\mathbf{X}$ be a point which neither coincides nor is contained in any of the base elements of $\mathbf{T}$, and let $\mathbf{X}^{\prime}=\mathbf{T}(\mathbf{X})$. Then,

1. the pencil of planes with axis $\mathbf{X} \vee \mathbf{B}_{1}$ maps to the pencil of planes with axis $\mathbf{X}^{\prime} \vee \mathbf{B}_{1}^{\prime}$.

2. the plane $\mathbf{X} \vee \mathbf{b}_{i}$ maps to the plane $\mathbf{X}^{\prime} \vee \mathbf{b}_{i}^{\prime}$, for $i=2,3$.

3. all other planes through $\mathrm{X}$ map to non-degenerate quadrics.

4. the line $\mathbf{X} \vee \mathbf{B}_{1}$ maps to the line $\mathbf{X}^{\prime} \vee \mathbf{B}_{1}^{\prime}$.

5. the pencil of lines with vertex $\mathbf{X}$ contained in the plane $\mathbf{X} \vee \mathbf{b}_{i}$ maps to the pencil of lines with vertex $\mathbf{X}^{\prime}$ contained in the plane $\mathbf{X}^{\prime} \vee \mathbf{b}_{i}^{\prime}$.

6. all other lines through $\mathbf{X}$ map to non-degenerate conics.

The proof can be found in [2], where it is also shown that the restriction of the space shape distortion to such preserved planes is a quadratic plane Cremona transformation. The structure of the planar transformation is similar to the space case, but its analysis is much simpler. Specifically, for a $C$-plane $\mathbf{p}$ (type 1 in Proposition 3), passing through $B_{1}$, the base elements of the planar shape distortion are three base points : $\mathbf{B}_{1}$, plus the two points $\mathbf{p} \wedge \mathbf{b}_{2}$ and $\mathbf{p} \wedge \mathbf{b}_{3}$. A quadratic plane Cremona transformation in general maps a line to a conic. The set of images of lines form the net of conics associated with the transformation. 
Similarly, $\mathbf{T}^{\prime}$ maps $\mathbf{f}_{1}^{\prime}$ onto $\mathbf{B}_{1}$, and each fundamental line of $\mathbf{f}_{2}^{\prime}\left(\mathbf{f}_{3}^{\prime}\right)$ to a point of $\mathbf{b}_{2}^{\prime}\left(\mathbf{b}_{3}^{\prime}\right)$.

Taken together, we call the base and the fundamental elements the singular elements of the Cremona transformation.

The Web of Quadrics A quadratic Cremona transformation maps a plane to a quadric surface. Thus, to the 3-parameter family of planes in true space there corresponds via $\mathbf{T}$ a 3 -parameter family of quadrics in apparent space. Similarly, $\mathbf{T}^{\prime}$ defines a 3-parameter family of quadrics in true space which are the images of planes in apparent space. Such a 3-parameter family is called a web of quadrics. An important property of the quadrics of a web is that each one of them contains the base elements of the associated quadratic Cremona transformation.

\subsection{Properties of the Shape Distortion}

In order to apply the global analysis to the shape distortion, the first step is to determine its singular elements. They are given in the following proposition :

Proposition 1. (Singular Elements of the Space Shape Distortion)

The singular elements of the space shape distortion $\mathbf{T}(\mathbf{X})$ (from $T h m .1$ ) are

$$
\begin{array}{lll}
\mathbf{B}_{1}=\mathbf{O}_{C} & \mathbf{b}_{2}=\mathbf{y}_{D} & \mathbf{b}_{3}=\mathbf{f}_{1} \wedge \mathbf{f}_{2} \\
\mathbf{f}_{1}=\mathbf{d}_{x}\left(\mathbf{O}_{C}^{\prime}\right) \mathbf{f}_{2}=\mathbf{c}\left(\mathbf{y}_{D}^{\prime}\right) & \mathbf{f}_{3}=\mathbf{B}_{1} \vee \mathbf{b}_{2},
\end{array}
$$

and the singular elements of its reverse $\mathbf{T}^{\prime}\left(\mathbf{X}^{\prime}\right)$ are

$$
\begin{array}{lll}
\mathbf{B}_{1}^{\prime}=\mathbf{O}_{C}^{\prime} & \mathbf{b}_{2}^{\prime}=\mathbf{y}_{D}^{\prime} & \mathbf{b}_{3}^{\prime}=\mathbf{f}_{1}^{\prime} \wedge \mathbf{f}_{2}^{\prime} \\
\mathbf{f}_{1}^{\prime}=\mathbf{d}_{x}^{\prime}\left(\mathbf{O}_{C}\right) & \mathbf{f}_{2}^{\prime}=\mathbf{c}^{\prime}\left(\mathbf{y}_{D}\right) \mathbf{f}_{3}^{\prime}=\mathbf{B}_{1}^{\prime} \vee \mathbf{b}_{2}^{\prime}
\end{array}
$$

The proof can be found in [2]. The proposition states several interesting facts :

- The dominant camera center $\mathbf{O}_{C}$ is the base point of the space shape distortion.

- The base conic splits into two base lines; one of them is $\mathbf{y}_{D}$, the $y$-axis of the non-dominant camera.

- The plane spanned by $\mathbf{O}_{C}$ and $\mathbf{y}_{D}$ is a plane of fundamental lines.

Since planes in general map onto quadrics passing through all three base elements, we can say that

Corollary 1. The shape distortion is particularly severe in the vicinity of the dominant camera center and the $Y$-axis of the non-dominant camera.

One can appreciate the severity of the distortion by imagining how the image of every plane must somehow converge to pass through the same base elements, for example the center of camera $C$. Thus, it is particularly important to take distortions into account in the near range of the stereo rig.

Our next result concerns changes in topology of a surface under the shape distortion : 


\section{Distortion of Curvature}

Our next task is to analyze the distortion of surface curvature. We first present results for the planar case, and then apply them to sections of surfaces by $C$ planes (type 1 in Proposition 3).

\subsection{The Planar Case}

A key result of the analysis of the planar shape distortion is the following :

Theorem 2. Let $\mathcal{C}$ be a planar curve through the point $\mathbf{X}$, with its image $\mathcal{C}^{\prime}$ under the planar shape distortion $\mathbf{T}$ passing through $\mathbf{X}^{\prime}=\mathbf{T}(\mathbf{X})$. Let $\kappa\left(\kappa^{\prime}\right)$ be the curvature of $\mathcal{C}\left(\mathcal{C}^{\prime}\right)$ at $\mathbf{X}\left(\mathbf{X}^{\prime}\right)$, and let $\kappa_{0}\left(\kappa_{0}^{\prime}\right)$ be the curvature of the net conic through $\mathbf{X}\left(\mathbf{X}^{\prime}\right)$ with the same tangent there as $\mathbf{C}\left(\mathbf{C}^{\prime}\right)$. Then, the curvatures of $\mathcal{C}$ and $\mathcal{C}^{\prime}$ are related as follows :

$$
\frac{\kappa^{\prime}}{\kappa_{0}^{\prime}}=1-\frac{\kappa}{\kappa_{0}}
$$

A proof of this can be found in [2]. From the curvature formula (12) we obtain a simple rule for the sign of the curvature of the distorted curve :

$$
\operatorname{sgn}\left(\kappa^{\prime}\right)=\left\{\begin{array}{cl}
\operatorname{sgn}\left(\kappa_{0}^{\prime}\right) & , \kappa<\kappa_{0} \\
0 & , \kappa=\kappa_{0} \\
-\operatorname{sgn}\left(\kappa_{0}^{\prime}\right) & , \kappa>\kappa_{0}
\end{array}\right.
$$

Thus, the curvature $\kappa^{\prime}$ changes sign when the curvature $\kappa$ of the original curve crosses the threshold $\kappa_{0}$ set by the curvature of the net conic. The relationship between the two curvatures is illustrated in Figure 3. The four curves through

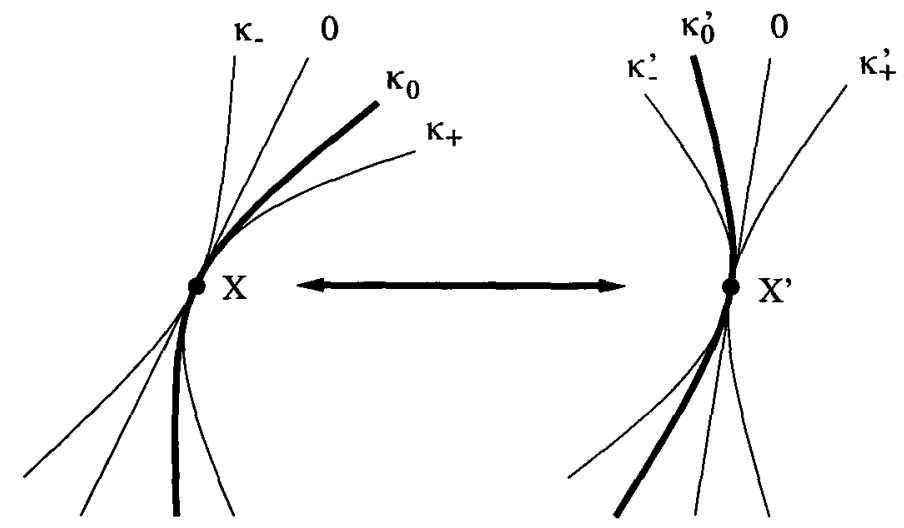

Fig. 3. Distortion of Curvature

$\mathbf{X}$ on the left map under $\mathbf{T}$ into four curves through $\mathbf{X}^{\prime}$ on the right. The 
curves are labelled by their curvatures, and form the following pairs under $\mathbf{T}$ : $\left\{\left(\kappa_{-}, \kappa_{-}^{\prime}\right),\left(0, \kappa_{0}^{\prime}\right),\left(\kappa_{0}, 0\right),\left(\kappa_{+}, \kappa_{+}^{\prime}\right)\right\}$. The thick curves belong to the net of conics defined by the quadratic transformation. The existence of the middle intervals in Figure 3 is what distinguishes quadratic from projective (linear) transformations. It reflects the fact that a convex curve may transform into a curve with a concave segment, i.e. that changes of convexity can occur.

\subsection{Distortion of Surface Curvature}

The local surface curvature at a point $\mathbf{X}$ on a surface depends on the direction in the tangent plane in which it is measured. Euler's formula[8] gives the normal curvature as a function of angle $\alpha$ in the tangent plane as :

$$
\kappa_{n}(\alpha)=\kappa_{1} \cos ^{2}\left(\alpha-\alpha_{1}\right)+\kappa_{2} \sin ^{2}\left(\alpha-\alpha_{1}\right)
$$

where $\kappa_{1}$ and $\kappa_{2}$ are the principal curvatures, i.e. the maximal and minimal normal curvatures at $\mathbf{X}$, and $\alpha_{1}$ is the angle corresponding to the direction associated with $\kappa_{1}$. The directions in which the principal curvatures occur are called principal directions. They are orthogonal. When $\kappa_{1}$ and $\kappa_{2}$ are of the same sign, the surface is locally elliptic, and when they are of opposite signs, the surface is hyperbolic. In the latter case there are locally two asymptotic directions in which the normal curvature vanishes. The asymptotic directions are bisectors of the principal directions.

If we take the section of a surface by a plane that is not normal to the surface, its curvature is given by Meusnier's Theorem $[8]: \kappa=\kappa_{n} / \cos \gamma$, where $\gamma$ is the angle between the sectioning plane and the normal to the surface at $\mathbf{X}$. By combining this with (14), we can determine the curvature of an arbitrary section of a surface.

Curvature of a Web Quadric In the planar case discussed in section 4.1, the curvature of a curve was compared to the curvature of the net conic to determine whether the convexity of the curve changes. Here, we relate the curvature of a given surface to the curvature of the web quadric with the same tangent plane. We do this by considering their section by the pencil of preserved planes with axis $\mathbf{O}_{C} \vee \mathbf{X}$.

We now outline a way in which the curvature of a web-quadric can be determined. But first, we derive some useful properties :

Proposition 4. The web quadrics associated with the space shape distortion and its reverse are hyperboloids of one sheet.

Proof. In section 3.1 we saw that every web quadric contains all base elements. In particular, every web quadric of $\mathbf{T}$ contains the two (intersecting) base lines $\mathbf{b}_{2}$ and $\mathbf{b}_{3}$, and every web quadric of $\mathbf{T}^{\prime}$ contains the two (intersecting) base line $\mathbf{b}_{2}^{\prime}$ and $\mathbf{b}_{3}^{\prime}$. The only non-degenerate quadrics that contain two intersecting lines are hyperboloids of one sheet [14]. 
A hyperboloid has the property that at each of its points the surface is locally hyperbolic, i.e. its principal curvatures are of opposite sign. Additionally, for a hyperboloid of one sheet the two lines through $\mathbf{X}$ in the asymptotic directions are contained as a whole in the surface[14]. They are its generators. The next proposition shows that they belong to the family of preserved lines discussed in Section 3.3.

\section{Proposition 5. (Generators of Web Quadrics)}

Let $\mathcal{Q}$ be a web quadric of the space shape distortion $\mathbf{T}$ at a point $\mathbf{X}$, and let $\mathbf{m}$ be its tangent plane there. Then, the two generators of $\mathcal{Q}$ through $\mathbf{X}$ are the lines of intersection $\mathbf{1}_{i}$ of $\mathbf{m}$ with the planes $\mathbf{X} \vee \mathbf{b}_{i}, i=2,3$.

Proof. The web quadric $\mathcal{Q}$ is the image under $\mathbf{T}^{\prime}$ of a plane $\mathbf{m}^{\prime}$. Consider the pencil of lines in $\mathbf{m}^{\prime}$ with vertex $\mathbf{X}^{\prime}$. Of the lines of this pencil only two are preserved as lines under $\mathbf{T}^{\prime}$, namely the lines $\mathbf{l}_{i}^{\prime}=\left(\mathbf{X}^{\prime} \vee \mathbf{b}_{i}^{\prime}\right) \wedge \mathbf{m}^{\prime}$ (type 5 in Proposition 3), which map to the lines $\mathrm{l}_{i}, i=2,3$. These two lines must therefore be the generators of $\mathcal{Q}$.

The construction of the generators described in the proposition is depicted in Figure 4. Since the directions in the tangent plane at $\mathbf{X}$ corresponding to

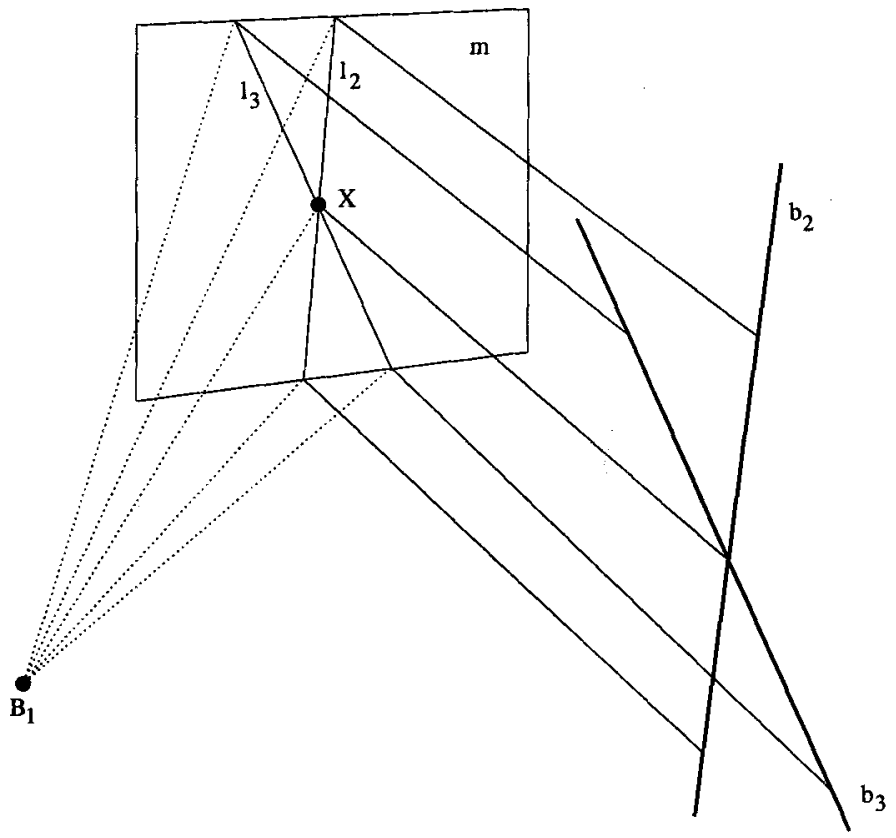

Fig. 4. The two generators $\mathbf{l}_{2}, \mathbf{l}_{3}$ through a point $X$ of a web quadric with tangent plane $\mathbf{m}$ at $\mathbf{X}$ are the lines of intersection of $\mathbf{m}$ with the two planes $\mathbf{X} \vee \mathbf{b}_{2}$ and $\mathbf{X} \vee \mathbf{b}_{3}$.

the generators are asymptotic directions, we can find the principal directions 


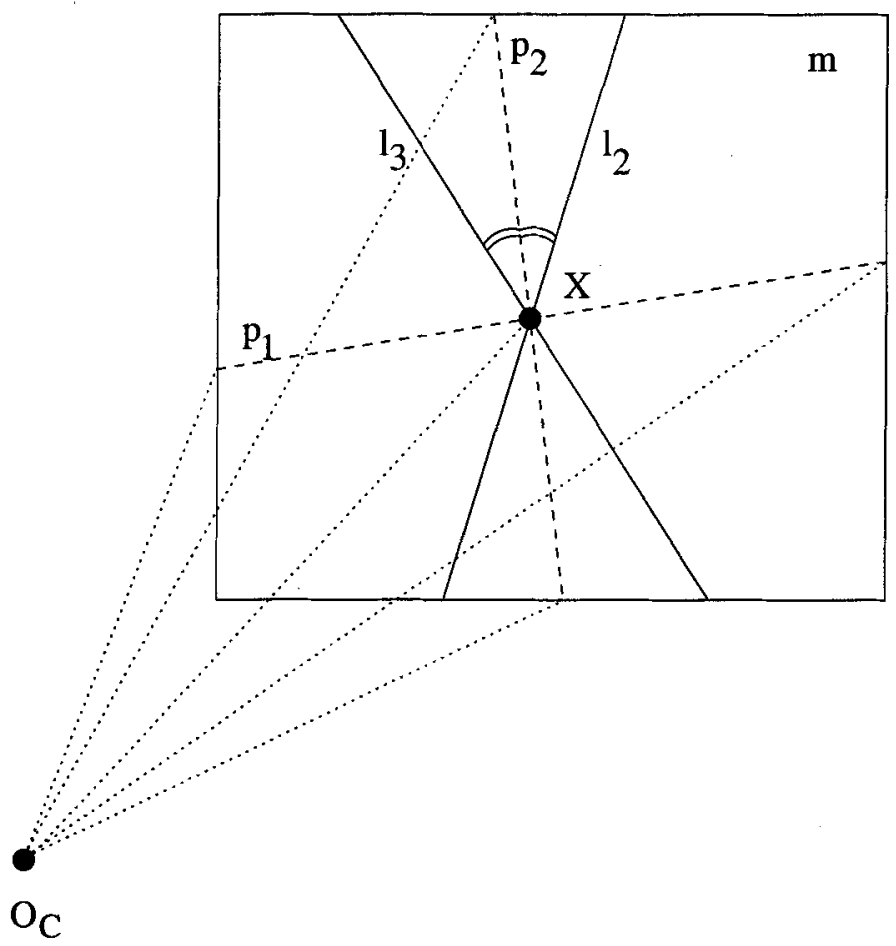

Fig. 5. The asymptotic directions in the tangent plane $m$ are along the generators $\mathrm{l}_{i}, i=2,3$, of the web quadric. The principal directions are bisectors of the asymptotic directions.

as their bisectors in $\mathbf{m}$, as mentioned above. This is shown in Figure 5. The principal curvatures of the web quadric can thus be obtained as follows : one computes the curvatures $\tilde{\kappa}_{1}$ and $\tilde{\kappa}_{2}$ of the sections of the web quadric by the two $C$-planes through the principal directions (indicated in Figure 5 by the dotted lines). Additionally, one computes the angles $\gamma_{1}$ and $\gamma_{2}$ between these $C$-planes and the normal to $\mathbf{m}$. Then, the principal curvatures of the web quadric are given by Meusnier's Theorem as $\kappa_{i}^{0}=\tilde{\kappa}_{i} / \cos \gamma_{i}$.

Qualitative Description of Curvature Distortion Let $\kappa_{1}$ and $\kappa_{2}$ denote the principal curvatures at a point $\mathbf{X}$ of a surface with tangent plane $\mathbf{m}$ at $\mathbf{X}$. Let $\alpha_{1}$ denote the principal direction associated with $\kappa_{1}$. The normal curvature of the surface is given by (14). Let $\alpha_{0}$ denote the principal direction associated with $\kappa_{1}^{0}$. The normal curvature of the web quadric is given by $\kappa_{0}(\alpha)=\kappa_{1}^{0} \cos ^{2}(\alpha-$ $\left.\alpha_{0}\right)+\kappa_{2}^{0} \sin ^{2}\left(\alpha-\alpha_{0}\right)$. The normal curvatures of the surface and of the web quadric are illustrated in Figure 6 (top figure). In each normal section, i.e. for each $\alpha$, the interval in which $\kappa(\alpha)$ lies determines the interval in which the 


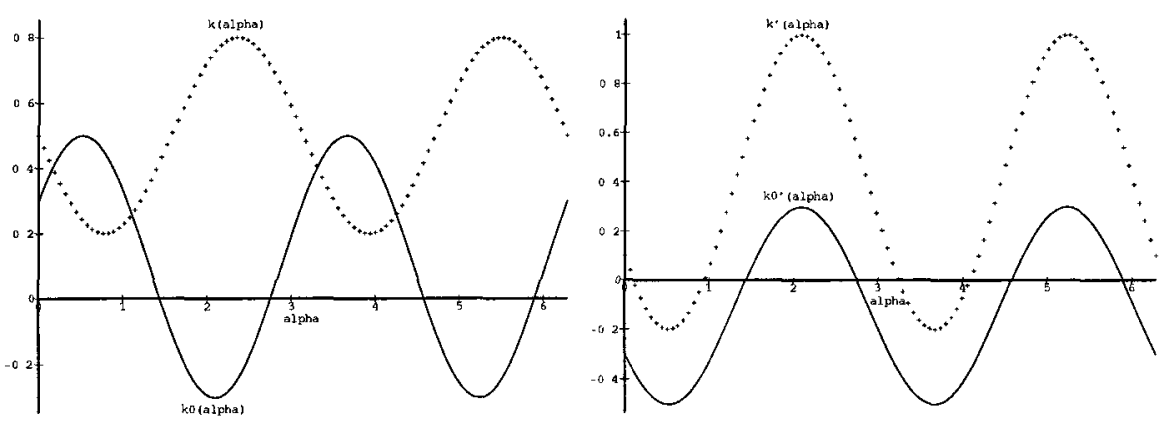

Fig. 6. Distortion of normal curvature in true space (left figure) and in apparent space (right figure). Solid curves represent the normal curvature of the web quadric as a function of direction. Dotted curves represent the normal curvature of the surface. See text for explanation.

distorted curvature $\kappa^{\prime}(\alpha)$ lies. For example, if $\kappa(\alpha)>\kappa_{0}(\alpha)$, then $\kappa^{\prime}(\alpha)>0$. For certain values of $\alpha$ the graph of $\kappa(\alpha)$ intersects the graph of $\kappa_{0}(\alpha)$. These are the values of $\alpha$ at which the sign of the distorted curvature changes. It should be emphasized that this can happen independently of whether the true normal curvature function ever passes through zero. A situation where the surface in the true space is locally elliptic (all normal curvatures positive), but the distorted surface is locally hyperbolic, is shown in Figure 6 . One sees there that the crossing of the graphs of $\kappa(\alpha)$ and $\kappa_{0}(\alpha)$ results in a zero-crossing of $\kappa^{\prime}(\alpha)$. This is the criterion under which an elliptic patch of a surface can transform to a hyperbolic patch on the distorted surface, and vice-versa.

\section{Conclusions}

We derived the shape distortion transformation for the general case of uncalibrated, and arbitrarily positioned and oriented, cameras, and showed that the full range of distortions for a stereo rig, due to errors in intrinsic and extrinsic camera parameters, is exactly modelled by quadratic space Cremona transformations.

Our analysis of the shape distortion transformation in terms of global properties of quadratic Cremona transformations suggested that the distortions are particularly severe in the vicinity of the camera centers.

Global properties of quadratic Cremona transformations further show that the topology and convexity of curves and surfaces can change under the shape distortion. We showed how violations of topology are governed by the crossing of certain planes defined in terms of the stereo geometry. Regarding violations 
of convexity, we derived the criterion for the change of surface type from elliptic to hyperbolic.

In this work we have explored the geometrical structure of the shape distortion. Future work will proceed along two directions. Firstly, we intend to perform a quantitative evaluation of the magnitude of the distortion for commonly encountered stereo geometries. Secondly, we intend to examine which tasks could be performed based on a distorted reconstruction of a scene, similarly to what has successfully been done for affine and projective reconstructions $[5,11]$.

\section{References}

1. G. Baratoff. Ordinal and metric structure of smooth surfaces from parallax. In Proc. Int. Conf. on Pattern Recognition ICPR'96, pages 275-279, 1996.

2. G. Baratoff. Distortion of stereoscopic visual space. Technical Report CAR-TR861, PhD Thesis, Center for Automation Research, University of Maryland, College Park, USA, May 1997.

3. G. Baratoff. Distortions of stereoscopic visual space and quadratic cremona transformations. In Proc. Computer Analysis of Images and Patterns CAIP'97, pages 239-246, 1997.

4. M. Barnabei, A. Brini, and G. Rota. On the exterior calculus of invariant theory. J. Algebra, 96:120-160, 1985.

5. P. A. Beardsley, A. Zisserman, and D. W. Murray. Navigation using affine structure from motion. In Proc. Europ. Conf. on Computer Vision ECCV'94, pages 85-96, 1994.

6. L. Cheong and Y. Aloimonos. Iso-distortion contours and egomotion estimation. In Proc. Int. Symp. on Computer Vision, pages 55-60, 1995.

7. O. Faugeras. What can be seen in three dimensions with an uncalibrated stereo rig. In Proc. Europ. Conf. on Computer Vision ECCV'92, pages 563-578, 1992.

8. E. Kreyszig. Differential Geometry. Dover Publications, New York, 1991.

9. Q. T. Luong and O. Faugeras. The fundamental matrix : Theory, algorithms, and stability analysis. Int. J. Computer Vision, 17:43-75, 1996.

10. S. Maybank. Theory of Reconstruction from Image Motion. Springer-Verlag, Berlin, 1993.

11. L. Robert and O. D. Faugeras. Relative $3 d$ positioning and $3 d$ convex hull computation from a weakly calibrated stereo pair. In Proc. Int. Conf. on Computer Vision ICCV'93, pages 540-543, 1993.

12. J.G. Semple and L. Roth. Introduction to Algebranc Geometry. Oxford University Press, London, 1949.

13. C. C. Slama, C. Theurer, and S. W. Henriksen. Manual of Photogrammetry. American Society of Photogrammetry, 1980.

14. B. Spain. Analytical Quadrics. Pergamon Press, Oxford, UK, 1960.

15. L. Svensson. On the use of double algebra in computer vision. Technical Report TRITA-NA-P9310, Swedish Royal Institute of Technology, Stockholm, 1993.

16. J. Weng, N. Ahuja, and T.S. Huang. Optimal motion and structure estimation. IEEE Trans. Pattern Analysis and Machine Intelligence, 15:864-884, 1993. 